\title{
Hybrid power control and contention window adaptation for channel congestion problem in internet of vehicles network
}

\author{
Hayder M. Amer ${ }^{1}$, Ethar Abduljabbar Hadi ${ }^{1}$, Lamyaa Ghaleb Shihab ${ }^{1}$, Hawraa H. Al Mohammed ${ }^{2}$, \\ Mohammed J. Khami ${ }^{1}$ \\ ${ }^{1}$ Department of Computer Systems, Technical Institute of Basrah, Southern Technical University, Basrah, Iraq \\ ${ }^{2}$ General Company for Ports of Iraq, Information Technology Department, Basrah, Iraq
}

\begin{abstract}
Article Info
ABSTRACT

Article history:

Received Jan 20, 2020

Revised Jun 26, 2021

Accepted Jul 19, 2021

Technology such as vehicular ad hoc networks can be used to enhance the convenience and safety of passenger and drivers. The vehicular ad hoc networks safety applications suffer from performance degradation due to channel congestion in high-density situations. In order to improve vehicular ad hoc networks reliability, performance, and safety, wireless channel congestion should be examined. Features of vehicular networks such as high transmission frequency, fast topology change, high mobility, high disconnection make the congestion control is a challenging task. In this paper, a new congestion control approach is proposed based on the concept of hybrid power control and contention window to ensure a reliable and safe communications architecture within the internet of vehicles network. The proposed approach performance is investigated using an urban scenario. Simulation results show that the network performance has been enhanced by using the hybrid developed strategy in terms of received messages, delay time, messages loss, data collision and congestion ratio.

Channel congestion

Contention window

IoV

Transmission power
\end{abstract}

This is an open access article under the CC BY-SA license.

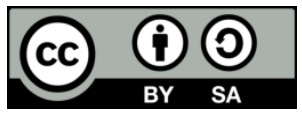

\section{Corresponding Author:}

Hayder M. Amer

Department of Computer Systems, Technical Institute of Basrah, Southern Technical University

Al-Zubeer Road, Basrah, Iraq

Email: hayder.amer@stu.edu.iq

\section{INTRODUCTION}

The main drive in the use of smart transportation systems is to reduce the number of vehicle accidents, enhance the efficiency of the transportation system, and enhance transportation safety. Smart cities employed internet of vehicles (IoV) that incorporate many communication systems such as vehicle to vehicle (V2V), vehicle to everything (V2X) and vehicle to infrastructure (V2I) which helps drivers to communicate and share traffic-related information [1]. In order to support the communication among V2X and V2I communication systems, the wireless access of vehicular environment (WAVE) [2] has been used by IoV. The IEEE 609 and the IEEE $802.11 \mathrm{p}$ protocols have been used to develop the medium access control (MAC) and physical layer of WAVE [3]. This facilitates the V2V and V2I systems to broadcast traffic information reaching short transmission areas.

Intelligent transportation systems utilize traffic information sharing among drivers by sending traffic messages through systems such as V2V and V2I. This helps to enhance the traffic mobility on the road maps and provide drivers with safe trips. The IoV channel congestion problem is deemed as a not trivial task. This is because it influences the accuracy of transmitted traffic information and the reliability of the network [4]. This dilemma happens when a vehicle broadcasts a high amount of information over the wireless channel of the network or when multiple nodes broadcast simultaneously many messages in a dense environment. The 
exiting of this dilemma related to other network factors such as nodes (vehicles) token bucket that is mean the number of arrival messages larger than the number of transmitting, the restricted channel capacity and fixed size of nodes buffer. The density of vehicles can grow linearly with the time which leads to creating high dense areas due to the vehicles traffic congestion. The traffic information can be disseminated several rates per second, the channel is predicted to become congested, degrading cooperative consciousness and intelligent transportation systems performance.

In order to avoid wireless link congestion in a case of increasing the density of vehicles in the coverage zone of a transmitting vehicle, the beacon interval of the vehicle will increase. Similarly, in case of low beacon reception rate or higher beacon loss rate, the transmitting frequency of beacon is decreased. In the case of the sparse network, rising the vehicle transmission spectrum leads to achieving the highest network closeness recognition. However, high network density with larger transmission range will burden the channel. To solve the overload in the channel during density network scenarios, the transmission power and the contention window adaptation schemes have been proposed. This paper develops a hybrid transmission power and contention window approach that is called PWCW to alleviate the wireless channel congestion problem in IoV networks. The paper novelty and main contributions are given as follows:

- To overcome the data channel congestion in IoV networks, we proposed a new channel congestion mitigation approach based on power control and contention window (PWCW). This approach adapting both the transmission power and contention window size of the wireless channel by considering the distances and densities of vehicles in the surrounding environments once the vehicle traffic congestion occurs. This helps to reduce the number of competing vehicles to access the channel and enhance the performance of the IoV networks.

- Increasing the contention window size leads to decreases interference rate; but, it harms the beacons broadcast delay. Therefore, in this paper it has been carefully considered by considering number of vehicles in the transmission range.

- The hybrid adaptive (PWCW) approach adjusts the transmission power and contention windows size of the transmitted messages. It also provides fairness in channel utilization. This helps to improve data dissemination and information accuracy.

- An overall performance assessment of the proposed approach is achieved in terms of received messages, delay time, messages loss, and data collision and congestion ratio. This assessment includes urban scenario testing. The results and discussion parts have shown that the developed protocol has better performance as compared to another protocol from literature. Improving the total number of received messages, reducing delay time to receive the traffic information, reducing the number of loss messages and decreasing the congestion among transmitted data helps to enhance the precision of the traffic information which in turn helps to design a better traffic management system.

The rest of the paper is structured as follows: The research method is given in section 2. Section 3 gives the formulation of the proposed method. The results and discussion are provided in section 4 . Lastly, in section 5 , conclusion is given.

\section{RESEARCH METHOD}

Discussions and investigations were broadly done with many approaches have been proposed for the congestion control problem in IoV. Traffic behaviour, network condition and/or parameters of the wireless channel which include (a 1-hope node density or number of neighbours, messages transmission rate, probability of received messages, nodes estimated distance, channel busy ratio, the quality of channel and number of packets loss) are used by adaptive beaconing approaches to adapt the transmission power, messages transmission rate, contention window size [5], [6]. As a result of their vital role on the channel congestion and communication overhead, this paper concentrates on the transmission power and contention window adaptation approaches.

Due to its high mobility, the link lifetime in IoV is limited and unpredictable which can be improved if a higher transmission power is deployed for vehicle communication. Thus, to decrease channel congestion in the vehicular networks, the power of transmitted messages for each vehicle needs to be adaptively regulated based on the network and channel characteristics. Adaptive transmission power affects the number of neighbouring vehicles that can receive beacon sent by their neighbours. Receiving/decoding correctly of the messages of low transmission power would just be allowed for the nearest neighbours. On the other hand, high power transmission would expand communication range enabling more neighbours that increase the number of the correctly received messages but increase interference among neighbour's nodes.

Nevertheless, due to the sending traffic information at a fixed rate and high topology change, adaptive power approaches can be employed to improve the network channel. Various adaptive beacon transmission power schemes have been suggested by researchers such as in [7]-[9]. Traffic density is used in 
the beacon power control scheme in [7] which is determined covering each vehicle as an essential foundation to modify power control. Increasing link connectivity over the congestion control in IoV was the focus of this scheme. Distributed fair power adjustment for vehicular networks (DFPAV) is proposed in [10]. Power distribution to every vehicle is obtained by extending the messages transmission power and maintaining every vehicle encountered messages load below the threshold value that is called maximum beaconing load (MBL).

Mittag et al. [11] are proposing two power control schemes known as segment-based power adjustment for vehicular environments (SPAV) and distributed vehicle density estimation (DVDE). The main goal of those two approaches is to reach maximum messages load with a low transmission power burden. Moreno et al. [12] propose the method of D-FPAV that is based on the dissemination of emergency messages in the IoV networks. This approach uses the concept of the water filling method to frequently designate the power for the transmitted safety messages for every vehicle. Lu and Poellabauer [13] has proposed an approach that is called Efficient transmit power control (ETPC) to increase the likelihood of the received messages at neighbouring vehicles at a desirable greatest broadcast spectrum. By piggybacking the power control information over the periodic beacons, the information of the specified power value is distributed among vehicles that are in the same transmission range. ETPC has high probability received messages as compared to the D-FPAV but at the cost of high channel overhead. The network topology p-persistent (NTPP) approach has been developed to consider the road traffic density to adapt the transmission power and guarantee the satisfactory coverage rate [14]. The particle swarm optimization method has been proposed in [8] to control the power of transmitted messages. This approach considers the adapting of the transmission power for constant messages sending rate by examining the channel state parameter that is called collision probability. This metric has been estimated by utilizing the number of surrounding vehicles and the number of received messages.

The power control MAC layer has been modelled as a game-theoretic approach in [15]. A model of Bayesian noncooperative game has been formulated to transmit traffic data for the communication bandwidth in deficient knowledge conditions. They analyzed the suggested approach as Bayesian Nash equilibrium, according to the channel gain based threshold the nodes transfer the beacon packet based on transmission power. They determine the achievement in terms of communication power prices and throughput compensations. Simulations have revealed that the game approach of the WAVE protocol gives a steady result once the channel is congested due to high transmission power. On the other hand, the transmission power and the contention window size $(\mathrm{CW})$ of MAC are other parameters that greatly affect the beacon collision.

The architecture is adaptively configuring the parameters of the MAC and network layers in a way the proposed approach improves MAC layer and messages properties to optimal rates for IoV [16]. Nevertheless, the dynamic density characteristics of IoV was not considered which are the density of vehicles variation between dense and sparse environment. A new CW value has been estimated to adaptive CW [17]. This approach is based on utilizing the density parameter of one-hop neighbour, the number of vehicles detected through an interval of $t$ and $\lambda$, where authors derived $\lambda$ through simulations. Several simulations had been performed to determine the relationship among the contention window, the density of vehicles, and the number of hidden nodes. The conclusion is the adaptation of the contention window can increase the probability of received messages and enhance network performance.

The MAC protocol was examined in [18], [19] to enhance broadcasting of beaconing messages. By dynamically adapting the contention windows to satisfy the best channel capacity requirements and weighted fairness. Zemouri et al. [19] evaluated the MAC performance in terms of reception probability by a periodic beacon message and each node's channel busy time during the broadcasting of a beacon. A hybrid beaconing approach has been proposed in [20], [21] to enhance the performance of transmitting traffic-related information in IoV network. The enhanced distribution of channel access (EDCA) mechanism has been utilized to identify various access categories (ACs) at the MAC layer to boost the transmission power at the physical (PHY) layer and offer the quality of service (QoS). The algorithm of the suggested heterogeneous adjustment transmission power and contention window was verified by designers employing simulations in ns-2 simulator. The average delay has been selected as a measurement criterion to evaluate performance and the results are compared with the standard IEEE 802.11 EDCA in [20], [21] with no CW adjustment. The results have shown that the developed approach has better performance and low delay variation.

As a result of the above schemes have been discussed, these approaches can reduce the delay, utilize the link fairly, increase the network proximity or link connectivity. Whereas, the schemes above fail to improve the IoV and target the main key challenges of the IoV at the same time. Thus, to utilize the available bandwidth efficiently, this paper proposes an approach when there is: i) a slight disconnection time due to the rapid change in the network topology and ii) data channel congestion due to the constant accumulation and propagation of traffic data. This proposed approach is a hybrid adaptive beaconing that provides the fairness in channel occupying which is accomplished by the adaptive of the transmission power and contention 
Window. Improving the accuracy of the information, the delay time of delivering the traffic information at every vehicle in the network is the main objective of the proposed hybrid adaptive power and contention. This approach helps to decrease messages loss rate and wireless channel congestion.

\section{THE PROPOSED METHOD}

In IoV, the data congestion occurs due to send messages at maximum transmission power to reach further distances, sending messages at high data rate and use small contention window size among competing nodes to occupying the channel. The congestion effects on the performance of the network and lead to high packets loss, which in turn reduces the accuracy of the transmitted data. In this paper, the hybrid approach has been proposed to adjust both the transmission power and contention window size to mitigate the channel congestion problem in the IoV.

\subsection{Transmission power adaptation}

Transmission power plays a major role in network congestion if the transmission power is high, its SNR will increase, but other SNR will decrease. Therefore, the transmission power has been selected based on the nearest node from the original transmitter. Figure 1 shows the mechanism of adapting transmission power in the proposed approach. Here, let assumes that there are 6 nodes around the central node $e_{0}$ and the transmission range of the Node $_{0}$ has been divided into four sectors as follows: Sector 1 has two nodes Node 1 and $\mathrm{Node}_{2}$, sector 2 has nodes number Node 3 and Node 4 sector 3 contains only Node 5 and sector number 4 includes node number Node $_{6}$.

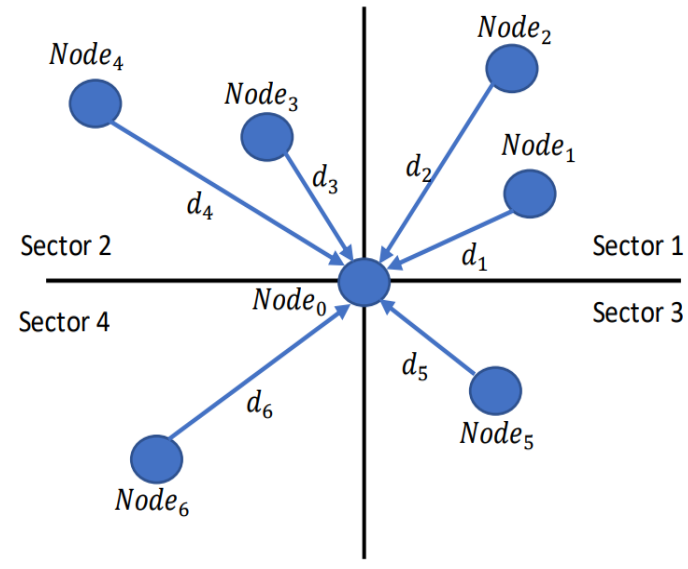

Figure 1. An example of estimating the maximum distance of nearest neighbour

In each sector, the nearest node has been selected based on the estimated distance $\left(d_{1}, d_{2}, d_{3}, d_{4}, d_{5}, d_{6}\right)$ from the central $N_{0} d_{0}$ that have been shown as in Figure 1. Each node will broadcast the beacon message every beacon period. In this message, vehicle position information is reflected. Therefore, neighbour nodes will compute the distance to source nodes. After the nearest node has been identified in each sector $\left(\right.$ Node $_{1}$, Node $_{3}, N_{o d e}$ and Node $e_{6}$ ). Then the maximum distance is selected among fours sector regarding nearest node $\left(d_{1}, d_{3}, d_{5}, d_{6}\right)$ and this distance is used to update the transmission power is according to the (1):

$$
P_{\text {loss }}=\frac{(4 \pi)^{2} d x^{\alpha}}{\lambda^{2}}
$$

where $P_{\text {loss }}$ the path loss model, $\mathrm{d}$ is is maximum distance that has been selected among fours sector regarding nearest node, $\alpha$ represents path loss component and $\lambda$ is the wavelength. The power transmission is updated by using (1) as (2):

$$
P_{t}=\frac{r_{s}}{P_{\text {loss }}}
$$


where $P_{t}$ is the next transmission power that vehicles will send messages with it, rs represents the receiver sensitivity which is given by the system designer.

\subsection{Contention window adaptation}

The contention window (CW) in the distributed coordination function of 802.11 uses the minimal value $C W_{\text {min }}$. Then the $\mathrm{CW}$ will be duplicated after each data collision until the maximum value is reached. The $C W_{\min }$ is reset to the initial value after each successful transmission without taking into account the density of vehicles in the crowded environment. This approach leads to an increase the congestion on the wireless channel due to competing many nodes to access the channel and selecting the same $C W_{\min }$ value which in turn degrades the network performance due to high data collision. This also increases the probability of packets loss.

Here, the $C W_{\min }$ has been adapted based on the number of vehicles. Each vehicle sends beacon messages periodically that contains information such as speed, the position and vehicle ID. These messages are utilized to estimate the number of contended vehicles in the transmission range. Two main parameters (contention evaluation and predicted offset) have been calculated to decrease competition and interference in a high-density environment. In the contention Evaluation, the number of neighbours of the sender vehicles is estimated based on utilizing the vehicle identification in the periodic beacon messages. Then each vehicle selects the predicted Offset slots by considering the determined number of neighbour vehicles.

The objective of the proposed approach is to diminish data collisions by adapting the transmission power and the $C W_{\min }$ size for every node. The $C W_{\min }$ is rigorously chosen based on the number of contesting vehicles. The new value of $C W_{\min }$ will boost utilization of the channel and increment number of received messages. The estimated $C W_{\min }$ value is raised linearly with increasing of vehicles number in the network environment. The adaptation of the $C W_{\min }$ has achieved a stable performance when compared to the constant $C W_{\min }$ size.

$$
E_{N}=\frac{\sum_{j=1}^{n} N j}{n}
$$

Where $E_{N}$ is the estimated number of contended vehicles. $N_{j}$ vehicle numbers that are participating in the transmission. Every vehicle determines the range of the offset slot provided the number of the competitor from equation 1 . Then, it determines the value of the required offset by using (2).

$$
\operatorname{Offset}_{N}=\frac{1}{N+1} \sum_{j=1}^{N} j
$$

Finally, the new $C W_{\min }$ is updated as (5):

$$
C W_{\text {min }_{\text {new }}}=\text { offset }_{N}+C W_{\text {min }_{\text {old }}}
$$

\section{RESULTS AND DISCUSSION}

The suggested approach has been examined and assessed by using the Veins [22], [23] that is a vehicular network simulator. Veins combines OMNeT++ network simulator [24] and the simulator for urban mobility (SUMO) [25], [26] to control movement and interaction among vehicular transmission modes. An urban road scenario has been created in SUMO and fed to the network simulator to test and measure the performance of the proposed approach as illustrated in Figure 2.

The suggested approach has been tested against various vehicle densities to evaluate its performance and effectiveness with the existing of the channel congestion. The PWCW has been analyzed and compared to another approach that is called power based Bayesian (PWBY) and it is implemented from literature as in [15]. Five types of network measurements have been used to evaluate the performance of the proposed approach as follows:

- Received packets (Mbps): It represents the total received messages at the receiver vehicle.

- Total delay time (ms): It is the total time required to deliver the messages from the transmitter to the receiver.

- Total packets loss: It indicates the number of lost messages due to channel congestion.

- Total data collisions: It represents the number of collision among transmitted data due to the channel occupied during the transmission. 


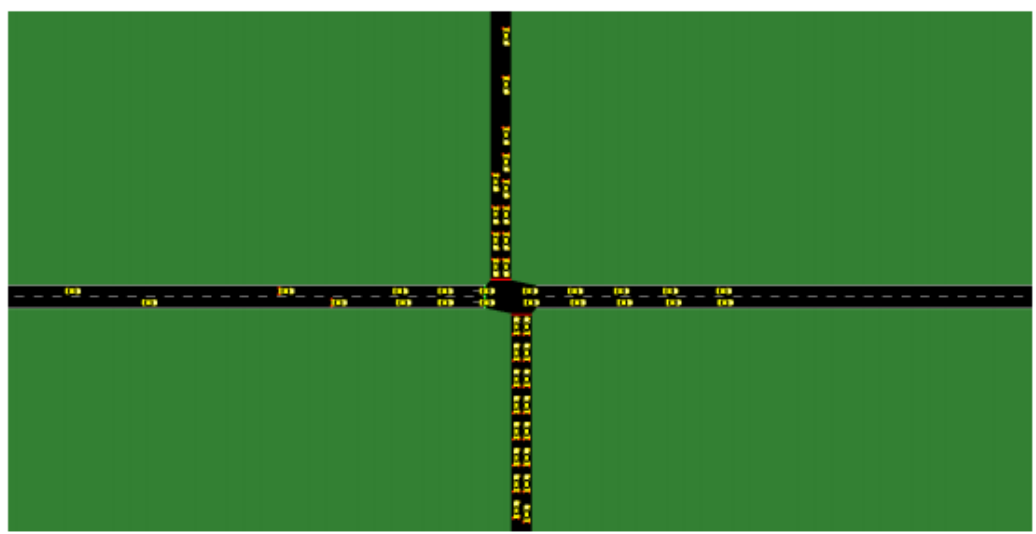

Figure 2. Veins simulation urban scenario

Table 1 presents the simulation parameters that have been chosen by the designer to evaluate and test the performance of the proposed approach. Figure 3 depicts the total number of received messages at the destination vehicles which is recorded by PWCW and PWBY, respectively. This figure has shown that the proposed approach has better delivery mechanism as compared to the PWBY. It is clear that when the congestion occurs in the network, the number of received messages is reduced due to packets loss and buffer overflow. However, the PWCW approach has significantly enhanced the total number of received messages at the destination vehicles. This is due to the effective adaptation of transmission power and a dynamic CWmin that is selected based on the density of vehicles which helps to reduce the number of lost messages as compared to the PWBY. The PWCW has adapted the power transmission by considering the nearest node from the original transmitter and then the maximum distance is chosen to update next transmission power that vehicles will send messages with it.

Table 1. The implemented scenario configuration parameters

\begin{tabular}{ccc}
\hline No. & Parameters & Values \\
\hline 1 & road mobility & Urban scenario \\
2 & Speed of nodes & $3.0-32 \mathrm{~m} / \mathrm{s}$ \\
3 & Vehicles density & $60,80,100,120,140,160$ \\
4 & Simulation time & $200 \mathrm{~s}$ \\
5 & Protocol & WAVE $(802.11 \mathrm{p})$ \\
6 & Transmission range & $400-1000$ \\
7 & Beacon frequency & 10 packets $/ \mathrm{s}$ \\
\hline
\end{tabular}

Figure 4 describes the total delay variation as compared to the density of the vehicles. It is obvious with the increase in the density of vehicles on the road segment, the time required to deliver messages is also increased which in turn boost delay variation. The result from the figure has shown that the PWCW has the lowest delay time as compared to the PWBY which has a sharp increment in the total delay time. This is due to the lack of the adaptation approach by considering only the transmission power in the formulated method, unlike the PWCW which has lower delay time in delivering the transmitted messages. This is due to consider two important parameters in the adaptation approach that are the power control of messages which is selected optimally based on the distance of the nearest node to the original sender and the adaptation of the CWmin has helped reduce the competition among the transmitted vehicles to access the wireless channel which in turn reduced the amount of time needed to deliver the messages.

Figure 5 demonstrates the effect of the channel congestion on the total amount of packets loss regarding the recorded results by PWCW and PWBY, respectively. The figure shows that the total amount of data that has been lost due to the channel congestion by PWCW is smaller than the amount recorded by PWBY. Figure 5 has shown that even with an increment of the density of the vehicles on the road segment the PWCW still has good performance in terms of packets loss, unlike the PWBY which increases the packets loss with the increments of the number of vehicles in the transmission area. This is due to employing an adaptive transmission power and dynamic CWmin which assists to alleviate the bottleneck in on the wireless channel. This minimizes the total loss packets, despite the density of the vehicles that are taken into the account. 


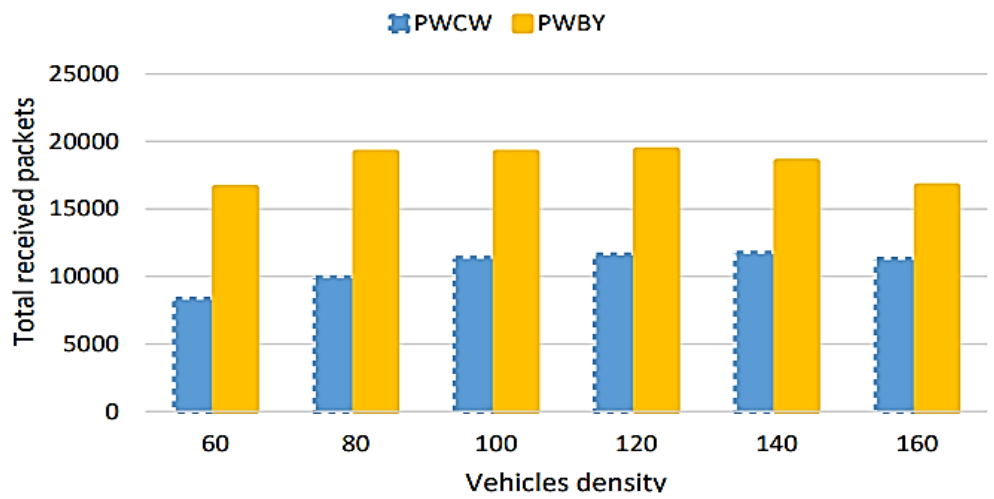

Figure 3. Total received messages

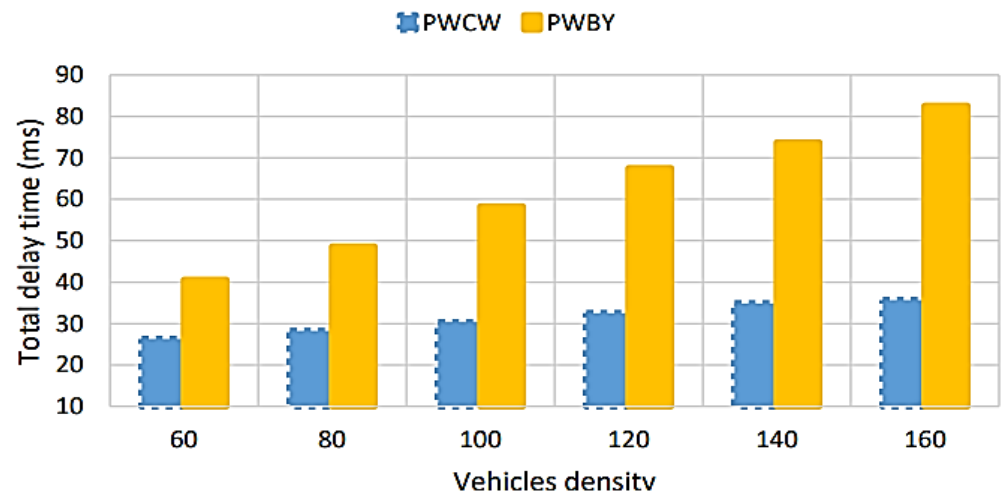

Figure 4. Total delay time

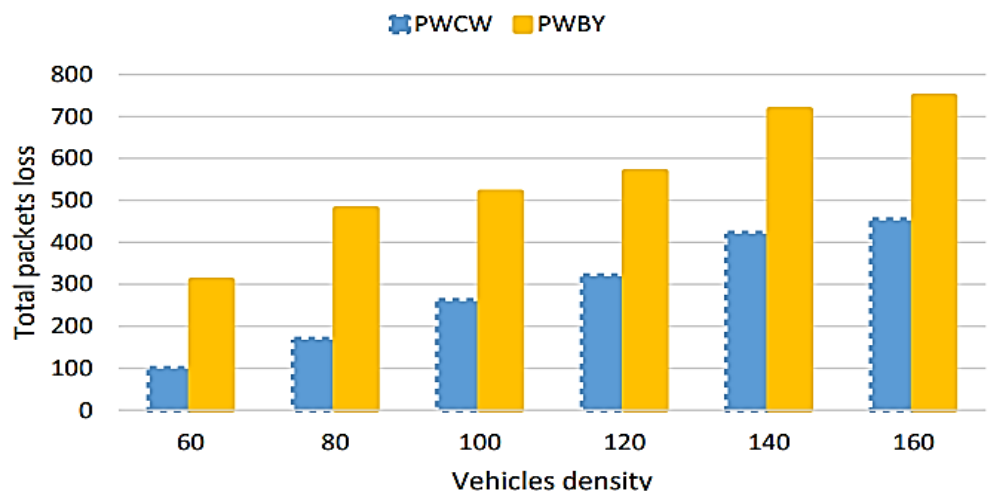

Figure 5. Total packets loss

Figure 6 describes the total data collision that is occurred due to exceed channel capacity and sending many messages over the channel at the same time. It is obvious that due to the considering multiple factors in the adaptation mechanism (transmission power and CWmin) by the PWCW, the number of collision in data has been reduced as compared to the total number recorded by the PWBY. The consequence of reducing total collision in data has been shown an effect and evident on the total amount of lost messages in Figure 5. The PWBY has a tremendous number of data collision. This is due to the competition among nodes attempting to transmit information with high information frequency and for further distance regardless of available resources.

Figure 7 reports the channel congestion ratio that is recorded by both PWCW and PWBY, respectively. It is clear that the quality of service parameters such as bandwidth, delay and packets loss has a 
huge effect on the result of the channel congestion ratio. The quality of service parameters has improved and the network performance has been enhanced by improving parameters such as number of received messages, delay time and number of messages loss. The PWCW has lower channel congestion ratio as compared to the PWBY approach. This is because two adaptation mechanisms has been considered in the proposed approach to alleviate the congestion on the wireless channel. PWBY has higher congestion ratio due to the lack of considering the density of the vehicles in the surrounding transmission range and relying on the static CWmin which in turn leads to increment the channel congestion.

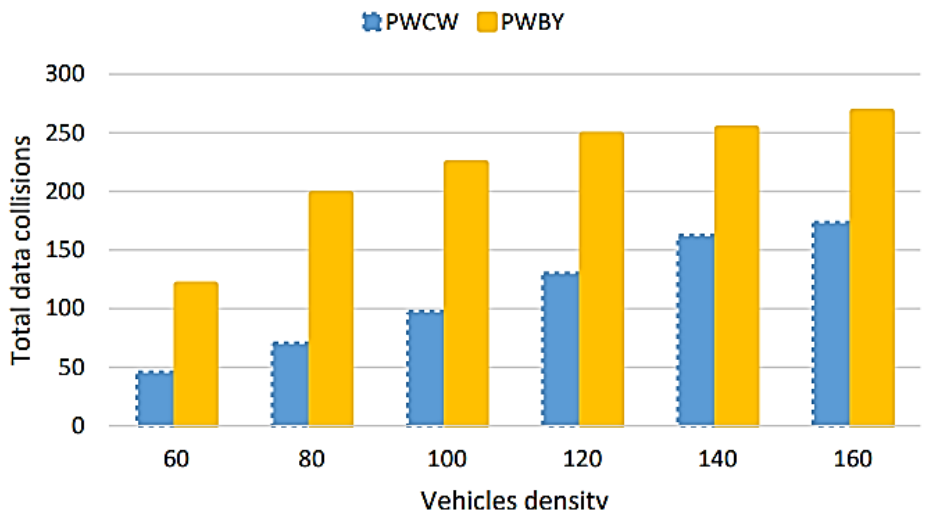

Figure 6. Total data collision

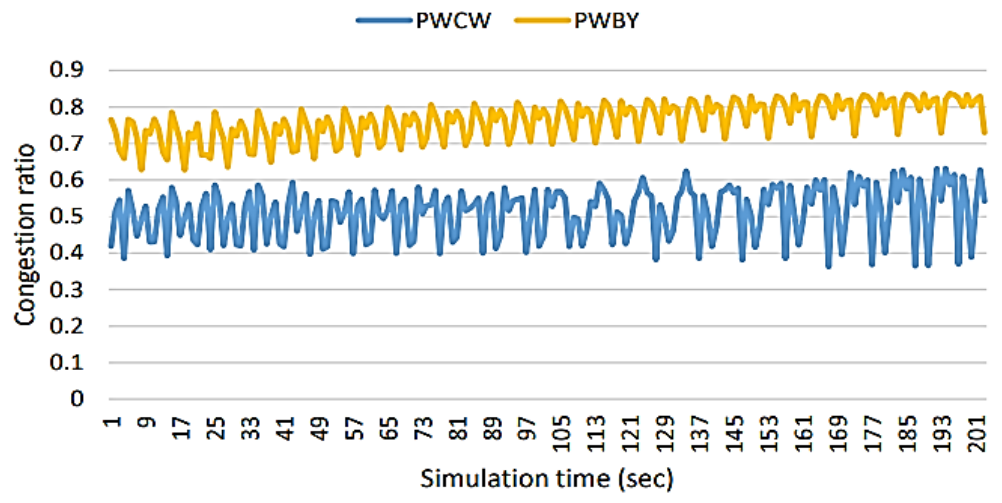

Figure 7. Channel congestion ratio

\section{CONCLUSION}

The channel congestion problem occurs in vehicular networks of the smart cities due to the increment in the density of the vehicles communicated on the road segments. The channel congestion significantly disturbs and reduces the performance of the network which in turn effects the communicated information among drivers which might be no longer valid due to the messages loss and delay variation. Therefore, this paper proposes a hybrid adaptation approach that is based on the transmission power adaptation and dynamic contention window size. This approach helps the network to reduce the node selfish behaviour by trying to send data at maximum power control. Simulation results confirm that the PWCW has a more reliable achievement in contrast to PWBY approach. The simulation results have illustrated that PWCW has a more reliable performance in contrast to the PWBY in terms of the total number of received messages at the destination node, lost messages, data collisions, delay variation and congestion ratio.

\section{ACKNOWLEDGEMENTS}

We are appreciative to support provided by the Ministry of Higher Education in Iraq. We are also grateful to the Southern Technical University- Technical Institute of BasraH for support. 


\section{REFERENCES}

[1] J. C. Castillo, S. Zeadally, and J. A. G. Ibañez, "Internet of vehicles: Architecture, protocols, and security," IEEE internet of things Journal, vol. 5, no. 5, pp. 3701-3709, 2017, doi: 10.1109/JIOT.2017.2690902.

[2] F. Lyu et al., "Characterizing urban vehicle-to-vehicle communications for reliable safety applications," IEEE Transactions on Intelligent Transportation Systems, vol. 21, no. 6, pp. 2586-2602, 2019, doi: 10.1109/TITS.2019.2920813.

[3] G. J. Sutton et al., "Enabling technologies for ultra-reliable and low latency communications: From phy and mac layer perspectives," IEEE Communications Surveys and Tutorials, vol. 21, no. 3, pp. 2488-2524, 2019, doi: 10.1109/COMST.2019.2897800.

[4] P. K. Singh, S. K. Nandi, and S. Nandi, "A tutorial survey on vehicular communication state of the art, and future research directions," Vehicular Communications, vol. 18, 2019, Art. no. 100164, doi: 10.1016/j.vehcom.2019.100164.

[5] X. Yang, L. Liu, N. H. Vaidya, and F. Zhao, "A vehicle-to-vehicle communication protocol for cooperative collision warning," in Proceedings of the First Annual International Conference on Mobile and Ubiquitous Systems: Networking and Services, 2004. MOBIQUITOUS 2004, 2004, pp. 114-123, doi: 10.1109/MOBIQ.2004.1331717.

[6] A. Rostami, B. Cheng, G. Bansal, K. Sjöberg, M. Gruteser, and J. B. Kenney, "Stability challenges and enhancements for vehicular channel congestion control approaches," IEEE Transactions on Intelligent Transportation Systems, vol. 17, no. 10, pp. 2935-2948, 2016, doi: 10.1109/TITS.2016.2531048.

[7] M. M. Artimy, W. Robertson, and W. J. Phillips, "Assignment of dynamic transmission range based on estimation of vehicle density," in Proceedings of the 2nd ACM international workshop on Vehicular ad hoc networks, 2005, pp. 40-48, doi: $10.1145 / 1080754.1080761$.

[8] G. Samara and T. Alhmiedat, "Intelligent emergency message broadcasting in vanet using PSO," World of Computer Science and Information Technology Journal (WCSIT), arXiv preprint arXiv: 1406.7399, vol. 4, no. 7, pp. 90-100, 2014.

[9] S. H. Bouk, G. Kim, S. H. Ahmed, and D. Kim., "Hybrid adaptive beaconing in vehicular ad hoc networks: A survey," International Journal of Distributed Sensor Networks, vol. 11, no. 5, 2015, doi: 10.1155\%2F2015\%2F390360.

[10] M. Torrent-Moreno, P. Santi, and H. Hartenstein., "Distributed fair transmit power adjustment for vehicular ad hoc networks," Proceedings of 3rd Annual IEEE Communications Society on Sensor and Ad Hoc Communications and Networks, vol. 2, 2006, pp. 479-488, doi: 10.1109/SAHCN.2006.288504

[11] J. Mittag, F. Schmidt-Eisenlohr, M. Killat, J. Härri, and H. Hartenstein, "Analysis and design of effective and low-overhead transmission power control for vanets," Proceedings of the fifth ACM international workshop on Vehicular Inter-Networking, 2008, pp. 39-48, doi: 10.1145/1410043.1410051.

[12] M. Torrent-Moreno, J. Mittag, P. Santi, and H. Hartenstein., "Vehicle-tovehicle communication: Fair transmit power control for safety-critical information," IEEE transactions on vehicular technology, vol. 58, no. 7, pp. 3684-3703, 2009, doi: 10.1109/TVT.2009.2017545.

[13] H. Lu and C. Poellabauer, "Balancing broadcast reliability and transmission range in vanets," 2010 IEEE Vehicular Networking Conference, 2010, pp. 247-254, doi: 10.1109/VNC.2010.5698263.

[14] K. A. Hafeez, L. Zhao, Z. Liaom, and B. N. W. Ma, "A new broadcast protocol for vehicular ad hoc networks safety applications," 2010 IEEE Global Telecommunications Conference GLOBECOM 2010, 2010, pp. 1-5, doi: 10.1109/GLOCOM.2010.5683409.

[15] Y. Kwon and B. Rhee, "Bayesian game-theoretic approach based on $802.11 \mathrm{p}$ mac protocol to alleviate beacon collision under urban vanets," International Journal of Automotive Technology, vol. 17, no. 1, pp. 183-191, 2016, doi: 10.1007/s12239-0160018-9.

[16] E. M. V. Eenennaam, G. Karagiannis, and G. Heijenk., "Towards scalable beaconing in vanets," Proceedings of Fourth ERCIM workshop on eMobility. Citeseer, 2010

[17] R. Stanica et al., "Broadcast communication in vehicular ad-hoc network safety applications," IEEE Consumer Communications and Networking Conference (CCNC), 2011, pp. 462-466, doi: 10.1109/CCNC.2011.5766513.

[18] J. Zhu and S. Roy, "Mac for dedicated short range communications in intelligent transport system," IEEE Communications Magazine, vol. 41, no. 12, pp. 60-67, 2003, doi: 10.1109/MCOM.2003.1252800.

[19] S. Zemouri, S. Djahel, and J. Murphy, "A fast, reliable and lightweight distributed dissemination protocol for safety messages in urban vehicular networks," Ad Hoc Networks, vol. 27, pp. 26-43, 2015, doi: 10.1016/j.adhoc.2014.11.016.

[20] D. B. Rawat et al., "Dynamic adaptation of joint transmission power and contention window in vanet," Proceedings of IEEE 70th Vehicular Technology Conference Fall, 2009, pp. 1-5, doi: 10.1109/VETECF.2009.5378793.

[21] D. B. Rawat, G. Yan, D. C. Popescu, M. C. Weigle, and S. Olariu., "Enhancing vanet performance by joint adaptation of transmission power and contention window size," IEEE Transactions on Parallel and Distributed Systems, vol. 22, no. 9, pp. 1528-1535, 2011, doi: 10.1109/VETECF.2009.5378793.

[22] C. Sommer, R. German, and F. Dressler, "Bidirectionally coupled network and road traffic simulation for improved IVC analysis," IEEE Transactions on Mobile Computing, vol. 10, no. 1, pp. 3-15, 2011, doi: 10.1109/TMC.2010.133.

[23] M. J. Haidari and Z. Yetgin, "Veins based studies for vehicular ad hoc networks," In 2019 International Artificial Intelligence and Data Processing Symposium (IDAP), 2019, pp. 1-7, doi: 10.1109/IDAP.2019.8875954.

[24] "What is OMNeT++?., OMNet++discrete event simulator, opensimltd. Web." omnetpp.org. https://omnetpp.org/ (accessed October 23, 2015)

[25] H. Yadav and C. N. Chandans, "Traffic congestion control using sumo simulator," International Scientific Journal of Contemporary Research in Engineering Science and Management, vol. 5, no. 2, pp. 211-215, 2020.

[26] P. A. Lopez et al., "Microscopic traffic simulation using sumo," 201821 st International Conference on Intelligent Transportation Systems (ITSC), 2018, pp. 2575-2582, doi: 10.1109/ITSC.2018.8569938. 